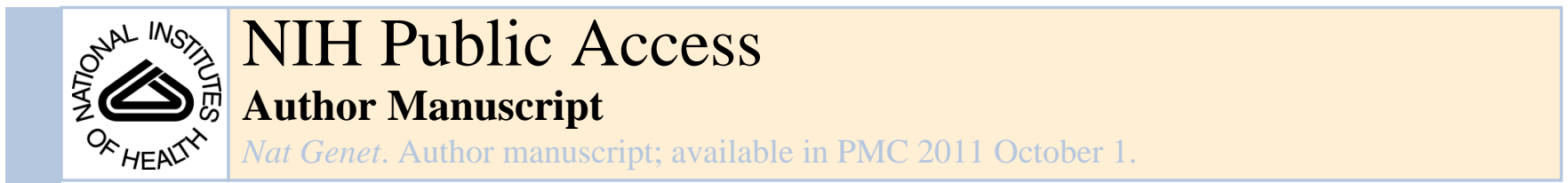

Published in final edited form as:

Nat Genet. ; 43(4): 333-338. doi:10.1038/ng.784.

\title{
Large-scale association analyses identifies 13 new susceptibility loci for coronary artery disease
}

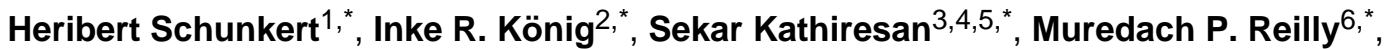
Themistocles L. Assimes ${ }^{7,{ }^{*}}$, Hilma Holm ${ }^{8,}{ }^{*}$, Michael Preuss ${ }^{1,2}$, Alexandre F. R. Stewart ${ }^{9}$, Maja Barbalic ${ }^{10}$, Christian Gieger ${ }^{11}$, Devin Absher ${ }^{12}$, Zouhair Aherrahrou ${ }^{1}$, Hooman Allayee $^{13}$, David Altshuler ${ }^{5,14}$, Sonia S. Anand ${ }^{15}$, Karl Andersen ${ }^{16,17}$, Jeffrey L. Anderson ${ }^{18}$, Diego Ardissino ${ }^{19}$, Stephen G. Ball ${ }^{20,21}$, Anthony J. Balmforth ${ }^{22}$, Timothy A. Barnes ${ }^{23}$, Diane M. Becker ${ }^{24}$, Lewis C. Becker ${ }^{24}$, Klaus Berger ${ }^{25}$, Joshua C. Bis ${ }^{26}$, S. Matthijs Boekholdt $^{27,28}$, Eric Boerwinkle ${ }^{10}$, Peter S. Braund ${ }^{23}$, Morris J. Brown ${ }^{29}$, Mary Susan Burnett $^{30}$, Ian Buysschaert ${ }^{31,32}$, Cardiogenics, John F. Carlquist ${ }^{18}$, Li Chen ${ }^{33}$, Sven Cichon $^{34,35,36}$, Veryan Codd ${ }^{23}$, Robert W. Davies ${ }^{37}$, George Dedoussis ${ }^{38}$, Abbas Dehghan ${ }^{39,40}$, Serkalem Demissie ${ }^{41,42}$, Joseph M. Devaney ${ }^{30}$, Ron Do ${ }^{43}$, Angela Doering ${ }^{11}$, Sandra Eifert ${ }^{44}$, Nour Eddine EI Mokhtari ${ }^{45}$, Stephen G. Ellis ${ }^{46}$, Roberto Elosua ${ }^{47}$, James C. Engert $^{43,48}$, Stephen E. Epstein ${ }^{30}$, Ulf de Faire ${ }^{49,50}$, Marcus Fischer ${ }^{51}$, Aaron R. Folsom ${ }^{52}$, Jennifer Freyer ${ }^{1}$, Bruna Gigante ${ }^{49,50}$, Domenico Girelli ${ }^{53}$, Solveig Gretarsdottir ${ }^{8}$, Vilmundur Gudnason $^{17,54}$, Jeffrey R. Gulcher ${ }^{8}$, Eran Halperin ${ }^{55,56,57}$, Naomi Hammond ${ }^{58}$, Stanley L. Hazen $^{59}$, Albert Hofman ${ }^{39}$, Benjamin D. Horne ${ }^{18}$, Thomas Illig $^{11}$, Carlos Iribarren ${ }^{60}$, Gregory T. Jones ${ }^{61}$, J.Wouter Jukema ${ }^{62,63}$, Michael A. Kaiser ${ }^{23}$, Lee M. Kaplan ${ }^{64}$, John J.P. Kastelein ${ }^{65}$, Kay-Tee Khaw ${ }^{66}$, Joshua W. Knowles ${ }^{7}$, Genovefa Kolovou ${ }^{67}$, Augustine Kong $^{8}$, Reijo Laaksonen ${ }^{68}$, Diether Lambrechts ${ }^{32}$, Karin Leander ${ }^{49}$, Guillaume Lettre ${ }^{69,70}$, Mingyao Li ${ }^{71}$, Wolfgang Lieb ${ }^{1}$, Patrick Linsel-Nitschke ${ }^{1}$, Christina Loley ${ }^{1,2}$, Andrew J. Lotery $^{72,73}$, Pier M. Mannucci ${ }^{74}$, Seraya Maouche ${ }^{1}$, Nicola Martinelli ${ }^{53}$, Pascal P.

Corresponding addresses: Prof. Dr. Heribert Schunkert, Universität zu Lübeck, Medizinische Klinik II, Ratzeburger Allee 160, 23538 Lübeck, Germany, Tel: + 49451500 2501, Fax: + 49451500 6437, heribert.schunkert@uk-sh.de, Prof. Nilesh J. Samani, Department of Cardiovascular Sciences, University of Leicester, Clinical Sciences Wing, Glenfield Hospital Groby Road, Leicester, LE3 9QP, United Kingdom, Tel: + 44116 2563021, Fax: + 44116 2875792, njs @le.ac.uk.

these authors contributed equally

AUTHORS CONTRIBUTIONS

Manuscript writing: H.S., I.R.K., S.K., M.P.R., T.L.A., H.H., A.F.R.S., P.D., R.R., R.M., J.E., N.J.S

GWA meta-analysis samples, genotyping and analysis: H.S., I.R.K., S.K., M.P.R., T.L.A., H.H., M.P., A.F.R.S., M.B., C.G., D.Absher, D.A., K.A., S.G.B., A.J.B., J.C.B., E.B., P.S.B., M.S.B., L.C., A.D., S.D., J.D., A.Doering, N.E.E.M., R.E., S.E., M.F., A.R.F, S.G., J.R.G., E.H., A.H., T.I., C.I., M.A.K., J.W.K., A.K., R.L., M.L., W.L., P.L.-N., C.L., C.M., T.M., O.M., V.M., K.M., T.M., J.N., C.P.N., A.P., L.Q., D.J.R., V.S., A.S., A.Schillert, S.S., J.S., S.M.S., D.S.S., K.S., G.Thorgeirsson, G.T., M.T., A.G.U., B.F.V., G.A.W., H.E.W., C.W., P.W., J.C.M.W., B.J.W.,T.Z., A.Z., F.C., L.A.C., T.Q., W.M., C.H., S.B., A.S.H., P.D., U.T., R.R., J.R.T., C.J.O’D., R.M., J.E., N.J.S.

Replication phase samples, genotyping and analysis: H.S., I.R.K., S.K., M.P.R., H.H., M.P., H.A., S.A., K.A., J.L.A., D.Ardissino, T.A.B., L.C.B, D.M.B., K.B., S.M.B., M.J.B., I.B., J.F.C., R.W.D., G.D., R.D., S.G.E., J.C.E., U.d.F., B.G., D.G., V.G., N.H., S.L.H., B.D.H., G.T.J., J.W.J., L.M.K., J.J.P.K., K.T.K., G.K., D.L., K.L., P.L.-N., A.J.L., P.M.M., N.M., P.P.M., P.A.M., T.Morgan, T.W.M., J.B.M., S.C., M.M.N., O.O., F.P., R.S.P., C.C.P., A.A.Q., L.S.R., F.R.R., D.R., M.L.S., M.S.S., M.S., S.Sivapalaratnam, A.V.S., T.B.S., J.D.S., N.S., J.A.S., K.Stark, K.S., M.Stoll, W.H.W.T., A.M.v.R., N.J.W., S.Y., P.D., U.T., R.R., R.M., J.E., N.J.S. Analysis group: I.R.K., M.P., D.Absher, L.C., E.H., M.L., K.M., A.Schillert, G.T., B.F.V., G.A.W., L.A.C., J.R.T.

Biological analyses: H.S., T.L.A., H.H., M.B., C.G., Z.A., P.S.B., V.C., J.F., S.G., P.L.-N., G.L., S.M., C.R., E.S., M.T., F.C.,

A.H.G., T.Q., C.H., W.H.O., P.D., U.T., J.E., N.J.S.

CARDIoGRAM consortium executive group: H.S., S.K., M.P.R., J.E., N.J.S

CARDIoGRAM consortium steering group: H.S., I.R.K., S.K., M.P.R., T.L.A., E.B., R.L., A.Z., C.H., A.S.H., U.T., J.R.T., R.M., J.E., N.J.S. Mooser are employees of GlaxoSmithKline. Hilma Holm, Solveig Gretarsdottir, Jeffrey R. Gulcher, Augustine Kong, Kari Stefansson, Gudmar Thorleifsson, Gudmundur Thorgeirsson, and Unnur Thorsteinsdottir are employees of and/or own stock/stock options in deCODE genetics. There are no other disclosures. 
McKeown ${ }^{75}$, Christa Meisinger ${ }^{11}$, Thomas Meitinger ${ }^{76,77}$, Olle Melander ${ }^{78}$, Pier Angelica Merlini $^{79}$, Vincent Mooser ${ }^{80}$, Thomas Morgan ${ }^{81}$, Thomas W. Mühleisen ${ }^{34,35}$, Joseph B. Muhlestein $^{18}$, Thomas Münzel ${ }^{82}$, Kiran Musunuru ${ }^{3,4,5}$, Janja Nahrstaedt ${ }^{1,2}$, Christopher P. Nelson $^{22}$, Markus M. Nöthen ${ }^{34,35}$, Oliviero Olivieri ${ }^{53}$, Riyaz S. Patel ${ }^{83,84}$, Chris C. Patterson ${ }^{75}$, Annette Peters ${ }^{11}$, Flora Peyvandi ${ }^{85}$, Liming Qu ${ }^{71}$, Arshed A. Quyyumi 83 , Daniel J. Rader ${ }^{6,86}$, Loukianos S. Rallidis ${ }^{87}$, Catherine Rice ${ }^{58}$, Frits R. Rosendaal ${ }^{88,89,90}$, Diana Rubin $^{91}$, Veikko Salomaa ${ }^{92}$, M. Lourdes Sampietro ${ }^{93}$, Manj S. Sandhu ${ }^{94,95}$, Eric Schadt $^{96,97}$, Arne Schäfer ${ }^{98}$, Arne Schillert ${ }^{2}$, Stefan Schreiber ${ }^{98}$, Jürgen Schrezenmeir ${ }^{99,100}$, Stephen M. Schwartz ${ }^{26}$, David S. Siscovick ${ }^{26}$, Mohan Sivananthan ${ }^{101}$, Suthesh Sivapalaratnam ${ }^{27}$, Albert Smith ${ }^{17,54}$, Tamara B. Smith ${ }^{102}$, Jaapjan D. Snoep ${ }^{88}$, Nicole Soranzo ${ }^{58}$, John A. Spertus ${ }^{103}$, Klaus Stark ${ }^{51}$, Kathy Stirrups ${ }^{58}$, Monika Stoll ${ }^{104}$, W. H. Wilson Tang ${ }^{46}$, Stephanie Tennstedt ${ }^{1}$, Gudmundur Thorgeirsson ${ }^{16,17}$, Gudmar Thorleifsson $^{8}$, Maciej Tomaszewski ${ }^{23,105}$, Andre G. Uitterlinden ${ }^{39,106,40}$, Andre M. van Rij ${ }^{61}$, Benjamin F. Voight $4,5,107$, Nick J. Wareham ${ }^{108}$, George A. Wells ${ }^{37}$, H.-Erich Wichmann $11,44,109$, Philipp S. Wild ${ }^{82}$, Christina Willenborg ${ }^{1,2}$, Jaqueline C. M. Witteman $^{39,40}$, Benjamin J. Wright ${ }^{110}$, Shu Ye ${ }^{111}$, Tanja Zeller ${ }^{82}$, Andreas Ziegler ${ }^{2}$, Francois Cambien $^{112}$, Alison H. Goodall ${ }^{23,105}$, L. Adrienne Cupples ${ }^{41,42}$, Thomas Quertermous ${ }^{7}$, Winfried März ${ }^{113,114,115}$, Christian Hengstenberg ${ }^{51}$, Stefan Blankenberg ${ }^{82}$, Willem H. Ouwehand $^{116,58}$, Alistair S. Hall ${ }^{11}$, Panos Deloukas ${ }^{58}$, John R. Thompson ${ }^{117}$, Kari

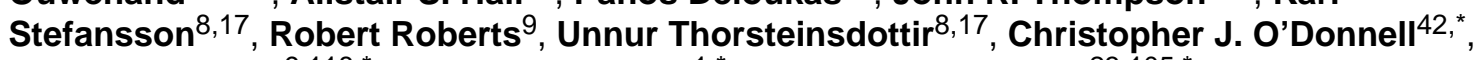

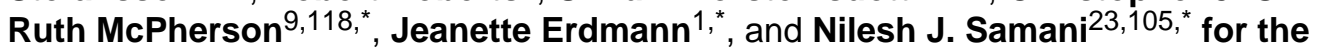
CARDIoGRAM Consortium

${ }^{1}$ Universität zu Lübeck, Medizinische Klinik II, Lübeck, Germany ${ }^{2}$ Institut für Medizinische Biometrie und Statistik, Universität zu Lübeck, Lübeck, Germany ${ }^{3}$ Cardiovascular Research Center and Cardiology Division, Massachusetts General Hospital, Boston, MA, USA ${ }^{4}$ Center for Human Genetic Research, Massachusetts General Hospital, Boston, MA, USA ${ }^{5}$ Program in Medical and Population Genetics, Broad Institute of MIT and Harvard, Cambridge, MA, USA ${ }^{6}$ The Cardiovascular Institute, University of Pennsylvania, Philadelphia, Pennsylvania 19104, USA ${ }^{7}$ Department of Medicine, Stanford University School of Medicine, Stanford, CA, USA ${ }^{8}$ deCODE Genetics, 101 Reykjavik, Iceland ${ }^{9}$ The John \& Jennifer Ruddy Canadian Cardiovascular Genetics Centre, University of Ottawa Heart Institute, Ottawa, Canada ${ }^{10}$ University of Texas Health Science Center, Human Genetics Center, Houston, TX, USA ${ }^{11}$ Institute of Epidemiology, Helmholtz Zentrum München, German Research Center for Environmental Health, Neuherberg, Germany ${ }^{12}$ Hudson Alpha Institute, Huntsville, Alabama, USA ${ }^{13}$ Department of Preventive Medicine University of Southern California Los Angeles, CA USA ${ }^{14}$ Department of Molecular Biology and Center for Human Genetic Research, Massachusetts General Hospital, Harvard Medical School, Boston, USA ${ }^{15}$ Population Health Research Institute, Hamiliton Health Sciences and McMaster University, Hamilton, Ontario, Canada ${ }^{16}$ Department of Medicine, Landspitali University Hospital, 101 Reykjavik, Iceland ${ }^{17}$ University of Iceland, Faculty of Medicine, 101 Reykjavik, Iceland ${ }^{18}$ Cardiovascular Department, Intermountain Medical Center; Cardiology Division, University of Utah. Salt Lake City, UT, USA ${ }^{19}$ Division of Cardiology, Azienda Ospedaliero-Universitaria di Parma, Parma, Italy ${ }^{20}$ LIGHT Research Institute, Faculty of Medicine and Health, University of Leeds, Leeds, UK ${ }^{21}$ Division of Cardiovascular and Neuronal Remodelling, Multidisciplinary Cardiovascular Research Centre, Leeds Institute of Genetics, Health and Therapeutics, University of Leeds, Leeds, UK ${ }^{22}$ Division of Cardiovascular and Diabetes Research, Multidisciplinary Cardiovascular Research Centre, Leeds Institute of Genetics, Health and Therapeutics, University of Leeds, Leeds, LS2 9JT, UK ${ }^{23}$ Department of Cardiovascular Sciences, University of Leicester, Clinical Sciences Wing, Glenfield Hospital, Leicester, LE3 9QP, UK ${ }^{24}$ The Johns Hopkins University School of Medicine, Division of General Internal Medicine, Baltimore, MD $21287{ }^{25}$ Institute of Epidemiology and Social Medicine, University of Münster, Germany ${ }^{26}$ Cardiovascular Health Resarch Unit and Department of 
Medicine, University of Washington, Seattle, WA USA ${ }^{27}$ Department of Vascular Medicine, Academic Medical Center, Amsterdam, The Netherlands ${ }^{28}$ Department of Cardiology, Academic Medical Center, Amsterdam, The Netherlands ${ }^{29}$ Clinical Pharmacology Unit, University of Cambridge, Cambridge, UK ${ }^{30}$ Cardiovascular Research Institute, Medstar Health Research Institute, Washington Hospital Center, Washington, DC 20010, USA ${ }^{31}$ Department of Cardiology, University Hospital Gasthuisberg, Leuven, Belgium ${ }^{32}$ Vesalius Research Center, VIB-KULeuven, Leuven, Belgium ${ }^{33}$ Cardiovascular Research Methods Centre, University of Ottawa Heart Institute, 40 Ruskin Street, Ottawa, Ontario, Canada, K1Y 4W7 ${ }^{34}$ Institute of Human Genetics, University of Bonn, Bonn, Germany ${ }^{35}$ Department of Genomics, Life \& Brain Center, University of Bonn, Bonn, Germany ${ }^{36}$ Institute of Neuroscience and Medicine (INM-1), Research Center Juelich, Juelich, Germany ${ }^{37}$ The Cardiovascular Research Methods, University of Ottawa Heart Institute, Ottawa, Ontario, Canada ${ }^{38}$ Department of Dietetics-Nutrition, Harokopio University, 17671 Athens, Greece ${ }^{39}$ Department of Epidemiology, Erasmus Medical Center, Rotterdam, The Netherlands ${ }^{40}$ Member of Netherlands Consortium for Healthy Aging (NCHA) sponsored by Netherlands Genomics Initiative (NGI), Leiden, The Netherlands ${ }^{41}$ Department of Biostatistics, Boston University School of Public Health, Boston, MA USA ${ }^{42}$ National Heart, Lung and Blood Institute's Framingham Heart Study, Framingham, MA, USA ${ }^{43}$ Department of Human Genetics, McGill University, Montreal, Canada ${ }^{44}$ Klinikum Grosshadern, Munich, Germany ${ }^{45} \mathrm{Klinik}$ für Innere Medizin, Kreiskrankenhaus Rendsburg, Rendsburg, Germany ${ }^{46}$ Department Cardiovascular Medicine, Cleveland Clinic, Cleveland, USA ${ }^{47}$ Cardiovascular Epidemiology and Genetics Group, Institut Municipal d'Investigació Mèdica, Barcelona; Ciber Epidemiología y Salud Pública (CIBERSP), Spain ${ }^{48}$ Department of Medicine, McGill University, Montreal, Canada ${ }^{49}$ Division of Cardiovascular Epidemiology, Institute of Environmental Medicine, Karolinska Institutet, Stockholm, Sweden ${ }^{50}$ Department of Cardiology, Karolinska University Hospital, Stockholm, Sweden ${ }^{51}$ Klinik und Poliklinik für Innere Medizin II, Universität Regensburg, Regensburg, Germany ${ }^{52}$ University of Minnesota School of Public Health, Division of Epidemiology and Community Health, School of Public Health (A.R.F.), Minneapolis, Minn.; USA ${ }^{53}$ Department of Medicine, University of Verona, Verona, Italy ${ }^{54}$ Icelandic Heart Association, Kopavogur Iceland ${ }^{55}$ The Blavatnik School of Computer Science, Tel-Aviv University, Tel-Aviv, Israel ${ }^{56}$ Department of Molecular Microbiology and Biotechnology, Tel-Aviv University, Tel-Aviv, Israel ${ }^{57}$ International Computer Science Institute, Berkeley, CA, USA ${ }^{58}$ Wellcome Trust Sanger Institute, Wellcome Trust Genome Campus, Hinxton, UK ${ }^{59}$ Lerner Research Institute, Cleveland Clinic, Cleveland, USA ${ }^{60}$ Division of Research, Kaiser Permanente of Northern California, Oakland, California, USA ${ }^{61}$ Surgery Department, Dunedin School of Medicine, University of Otago, New Zealand ${ }^{62}$ Department of Cardiology C5-P, Leiden University Medical Center, Leiden, The Netherlands ${ }^{63}$ Durrer Center for Cardiogenetic Research, Amsterdam, The Netherlands ${ }^{64}$ Massachusetts General Hospital, Boston MA, USA ${ }^{65}$ Dept. Vascular Medicine, Academic Medical Center, University of Amsterdam, Amsterdam, The Netherlands ${ }^{66}$ Department of Public Health and Primary Care, Strangeways Research Laboratory, University of Cambridge, Cambridge, UK ${ }^{67} 1$ st Cardiology Department, Onassis Cardiac Surgery Center, 356 Sygrou Avenue, 17674 Athens, Greece ${ }^{68}$ Science Center, Tampere University Hospital, Tampere, Finland ${ }^{69}$ Montreal Heart Institute, Montréal, Québec, H1T 1C8, Canada ${ }^{70}$ Département de Médecine, Université de Montréal, C.P. 6128, succursale Centre-ville, Montréal, Québec, H3C 3J7, Canada ${ }^{71}$ Biostatistics and Epidemiology, University of Pennsylvania, Philadelphia, PA, USA ${ }^{72}$ Clinical Neurosciences Division, School of Medicine, University of Southampton, Southampton, UK ${ }^{73}$ Southampton Eye Unit, Southampton General Hospital, Southampton, UK ${ }^{74}$ Scientific Direction, IRCCS Fondazione Cà Granda, Ospedale Maggiore Policlinico, Milano, Italy ${ }^{75}$ Centre for Public Health, Queen's University Belfast, Institute of Clinical Science, Belfast, Northern Ireland, UK ${ }^{76}$ Institute of Human Genetics, Helmholtz Zentrum München, Deutsches Forschungszentrum für Umwelt und Gesundheit, Neuherberg, Germany ${ }^{77}$ Institute of Human Genetics, Technische Universität München, Klinikum rechts der Isar, Munich, Germany 
${ }^{78}$ Department of Clinical Sciences, Hypertension and Cardiovascular Diseases, Scania University Hospital, Lund University, Malmö, Sweden ${ }^{79}$ Division of Cardiology, Azienda Ospedaliera Niguarda Ca'Granda, Milan, Italy ${ }^{80}$ Genetics Division and Drug Discovery, GlaxoSmithKline, King of Prussia, Pennsylvania 19406, USA ${ }^{81}$ Department of Pediatrics, Vanderbilt University School of Medicine, Nashville ${ }^{82}$ 2. Medizinische Klinik und Poliklinik, Universitätsmedizin Mainz, JohannesGutenberg Universität Mainz, Germany ${ }^{83}$ Emory University School of Medicine, Atlanta GA, USA ${ }^{84}$ Cardiff University, Cardiff, Wales, UK CF10 3XQ ${ }^{85}$ A. Bianchi Bonomi Hemophilia and Thrombosis Center, Department of Medicine and Medical Specialties, Fondazione IRCCS Ca Granda, Ospedale Maggiore Policlinico, Università degli Studi di Milano and Luigi Villa Foundation, Milan, Italy ${ }^{86}$ The Institute for Translational Medicine and Therapeutics, School of Medicine, University of Pennsylvania, Philadelphia, PA, USA ${ }^{87}$ Second Department of Cardiology, Attikon Hospital, School of Medicine, University of Athens, Athens, Greece ${ }^{88}$ Department of Clinical Epidemiology, Leiden University Medical Center, Leiden, The Netherlands ${ }^{89}$ Department of Thrombosis and Haemostasis, Leiden University Medical Center, Leiden, The Netherlands ${ }^{90}$ Einthoven Laboratory for Experimental Vascular Medicine, Leiden University Medical Center, Leiden, The Netherlands ${ }^{91}$ Medizinische Klinik I, Universitätsklinikum Schleswig-Holstein, Campus Kiel, Kiel, Germany ${ }^{92}$ Chronic Disease Epidemiology and Prevention Unit, Department of Chronic Disease Prevention, National Institute for Health and Welfare, Helsinki, Finland ${ }^{93}$ Department of Human Genetics and Cardiology, Leiden University Medical Center, Leiden, the Netherlands ${ }^{94}$ Manjinder S Sandhu, Genetic Epidemiology Group, Wellcome Trust Sanger Institute, Cambridge, UK ${ }^{95}$ Department of Public Health \& Primary Care, Strangeways Research Laboratory, University of Cambridge, UK ${ }^{96}$ Pacific Biosciences, 1505 Adams Drive, Menlo Park, CA $94025{ }^{97}$ Sage Bionetworks, Palo Alto, CA $94301{ }^{98}$ Institut für Klinische Molekularbiologie, Christian-Albrechts Universität, Kiel, Germany ${ }^{99}$ Institute of Physiology and Biochemistry of Nutrition, Max Rubner-Institute, Kiel, Germany ${ }^{100}$ Clinical Research Center Kiel, Kiel Innovation and Technology Center, Kiel, Germany ${ }^{101}$ Cardiology Division, Leeds Teaching Hospitals NHS Trust, Leeds, UK ${ }^{102}$ Laboratory of Epidemiology, Demography, and Biometry, Intramural Research Program, National Institute on Aging, National Institutes of Health, Bethesda MD, USA ${ }^{103}$ Mid America Heart Institute and University of Missouri-Kansas City, Kansas City ${ }^{104}$ LeibnizInstitute for Arteriosclerosis Research, University of Münster, Münster, Germany ${ }^{105}$ Leicester National Institute for Health Research Biomedical Research Unit in Cardiovascular Disease, Glenfield Hospital, Leicester, LE3 9QP, UK ${ }^{106}$ Department of Internal Medicine, Erasmus Medical Center, Rotterdam, The Netherlands ${ }^{107}$ Department of Medicine, Harvard Medical School, Boston, MA, USA ${ }^{108}$ MRC Epidemiology Unit, Institute of Metabolic Science, Addenbrooke's Hospital, Cambridge, UK ${ }^{109}$ Institute of Medical Information Science, Biometry and Epidemiology, Ludwig-Maximilians-Universität München, Germany ${ }^{110}$ Department of Cardiovascular Surgery, University of Leicester, Leicester, UK ${ }^{111}$ William Harvey Research Institute, Barts and The London School of Medicine and Dentistry, Queen Mary University of London, London, UK ${ }_{112}$ INSERM UMRS 937, Pierre and Marie Curie University, UPMC-Paris 6, Faculté de Médecine Pierre et Marie Curie, Paris, France ${ }^{113}$ Synlab Center of Laboratory Diagnostics Heidelberg, Heidelberg, Germany ${ }^{114}$ Clinical Institute of Medical and Chemical Laboratory Diagnostics, Medical University of Graz, Graz, Austria ${ }^{115}$ Institute of Public Health, Social and Preventive Medicine, Medical Faculty Manneim, University of Heidelberg, Germany ${ }^{116}$ Department of Haematology, University of Cambridge and NHS Blood and Transplant, Cambridge, UK ${ }^{117}$ Department of Health Sciences, University of Leicester, Leicester, UK ${ }^{118}$ Atherogenomics Laboratory, University of Ottawa Heart Institute, Ottawa, Canada

\section{Abstract}

We performed a meta-analysis of 14 genome-wide association studies of coronary artery disease (CAD) comprising 22,233 cases and 64,762 controls of European descent, followed by genotyping of top association signals in 60,738 additional individuals. This genomic analysis identified 13 
novel loci harboring one or more SNPs that were associated with CAD at $P<5 \times 10^{-8}$ and confirmed the association of 10 of 12 previously reported CAD loci. The 13 novel loci displayed risk allele frequencies ranging from 0.13 to 0.91 and were associated with a 6 to 17 percent increase in the risk of CAD per allele. Notably, only three of the novel loci displayed significant association with traditional CAD risk factors, while the majority lie in gene regions not previously implicated in the pathogenesis of CAD. Finally, five of the novel CAD risk loci appear to have pleiotropic effects, showing strong association with various other human diseases or traits.

It has been estimated that heritable factors account for 30-60\% of the interindividual variation in the risk of coronary artery disease $(\mathrm{CAD})^{1}$. Recently, genome-wide association (GWA) studies have identified several common variants that associate with risk of CAD ${ }^{2}$. However, in aggregate these variants explain only a small fraction of the heritability of CAD, probably partly due to the limited power of previous studies to discover effects of modest size. Recognizing the need for larger studies, we formed the transatlantic Coronary ARtery DIsease Genome wide Replication and Meta-analysis (CARDIoGRAM) consortium ${ }^{3}$. We perfomed a meta-analysis of 14 GWA studies of CAD comprising 22,233 cases and 64,762 controls, all of European ancestry (Supplementary Table 1a and Additional Table 1a, see www.imbs-luebeck.de/imbs/sites/default/files/myfilemanager/500/

AdditionalInformation.pdf). We then genotyped lead SNPs within the most promising novel loci as well as a subset of previously reported CAD loci in up to 60,738 additional subjects (approximately half cases and controls) (Supplementary Table 1b and Additional Table 1b, see www.imbs-luebeck.de/imbs/sites/default/files/myfilemanager/500/

AdditionalInformation.pdf). Lastly, we explored potential mechanisms and intermediate pathways by which novel loci may mediate risk.

Nine of the 12 loci previously associated with CAD through individual GWA studies achieved genome-wide significance $\left(P<5 \times 10^{-8}\right)$ in our initial meta-analysis (Table 1$)$. We were, however, unable to test the previously reported association with a haplotype and a rare SNP in $L P A$ in our GWA data, ${ }^{4-5}$ but observed robust association with the rare $L P A$ variant in our replication samples through direct genotyping (Table 1). Thus, 10 of the 12 loci previously associated with $\mathrm{CAD}$ at a genome-wide significance level surpassed the same threshold of significance in CARDIoGRAM.

We selected 23 novel loci with a significance level of $P<5 \times 10^{-6}$ in the meta-analysis for follow-up (see Online Methods and Supplementary Note for details). Taking the number of loci into consideration, our replication study had $>90 \%$ power to detect effect sizes observed in the GWA meta-analysis. Of the 23 loci, 13 replicated using our a priori definition of a validated locus i.e., showing independent replication after Bonferroni correction and also achieving a $\mathrm{P}$ value of $<5 \times 10^{-8}$ in the combined discovery and replication data (Table 2, Figure 1, Supplementary Figures 1 and 2). Results for all loci in the replication phase are shown in Supplementary Table 2.

The 13 novel loci displayed risk allele frequencies ranging from 0.13 to 0.91 and were associated with a 6 to 17 percent increase in the risk of CAD per allele (Table 2). Out of the 13 novel loci the additive model appeared most appropriate for six while the recessive model performed best at 5 and the dominant model at 2 loci (Additional Table 2, see www.imbs-luebeck.de/imbs/sites/default/files/myfilemanager/500/ AdditionalInformation.pdf).

In sub-group analyses, 20 out of 22 loci with $P<5 \times 10^{-8}$ (known and novel loci combined; for one locus age subgroups were not available) had higher odds ratios for early-onset than for late onset CAD ( $P=1.2 \times 10^{-4}$ for observed vs. expected, Supplementary Table 3$)$. The 
CAD loci showed consistent associations irrespective of case definition, although the odds ratios for most individual single nucleotide polymorphisms (SNPs) tended to be slightly greater for cases with angiographically proven CAD than for cases with unknown angiographic status $(P=0.019$ for observed vs. expected). In contrast, sub-group analyses in males and females revealed no sex specific effects for any risk alleles (Supplementary Table 3 ) or for their observed vs. expected pattern of association $(P=0.4)$.

Among 7,523 controls and 7,637 CAD cases for whom we had individual-level genotype data, the minimum and maximum number of risk alleles observed per individual was 15 and 37 , respectively, when considering $23 \mathrm{CAD}$ susceptibility loci. The mean weighted risk score was significantly higher for cases than for controls $\left(P<10^{-20}\right)$. Furthermore, being in the top $10^{\text {th }}$ percentile or lowest $10^{\text {th }}$ percentile of the weighted score was associated with an odds ratio for CAD of 1.88 (95\% confidence interval, 1.67 to 2.11 ) and 0.55 (95\% confidence interval, 0.48 to 0.64 ), respectively, compared to the $50^{\text {th }}$ percentile. The change in odds ratio for CAD across a broader spectrum of categories of the weighted score is shown in Supplementary Figure 3.

Three of the novel risk alleles were associated with differences in traditional CAD risk factors (Table 3 and Supplementary Table 4). The risk allele on chromosome 11q23.3 (rs964184, ZNF259, APOA5-A4-C3-A1 gene region) was associated with increased LDL cholesterol and decreased HDL cholesterol (and previously, with increased triglycerides) ${ }^{6}$. The risk allele on chromosome 9q34.2 (rs579459, ABO) was associated with increased LDL and total cholesterol, in a direction consistent with the association of these SNPs with CAD risk (Table 3). The variant rs12413409 on chromosome 10q24.32 representing the CYP17A1/CNNM2/NT5C2 gene region was associated with hypertension.

In silico interrogation revealed that the lead SNPs at four of the 13 novel loci were either non-synonymous coding variants or were in high LD with such SNPs. Specifically, the lead SNPs at 7q32.2 (rs11556924) and 15q25.1 (rs3825807) encoded changes in ZC3HC1 (R363H) and ADAMTS7 (S214P), respectively, while the lead SNP at 14q32.2 (rs2895811) is in strong LD $\left(r^{2}=0.82\right)$ with the V691A in the HHIPL1 gene. Lastly, the lead SNP at $17 \mathrm{q} 21.32$ (rs46522) is in strong $\mathrm{LD}\left(r^{2}=0.94\right)$ with two potential functional variants in GIP: S103G (rs2291725) and a variant influencing the splice site of intron 3 (rs2291726) leading to a truncated transcript (Additional Table 3, see www.imbs-luebeck.de/imbs/sites/default/files/myfilemanager/500/ AdditionalInformation.pdf) ${ }^{7}$.

We next analyzed data from three genome-wide studies that also assessed gene expression in multiple tissues to assess potential effects of novel loci on the expression of regional genes (Supplementary Note) $^{8-9}$. Three of the novel CAD risk variants showed convincing association with regional gene expression (cis effect) by either representing the most significant eSNP in the region or by being in high LD $\left(\mathrm{r}^{2} \geq 0.85\right)$ with the strongest eSNP in the region: rs12190287 at 6q23.2 (TCF21), rs12936587 at 17p11.2 (RASD1, SMCR3 and $P E M T)$ and rs46522 at 17q21.32 (UBE2Z) (Additional Table 4, see www.imbs-luebeck.de/imbs/sites/default/files/myfilemanager/500/ AdditionalInformation.pdf). Subsequent interrogation of our novel loci in a genome-wide map of allelic expression imbalance provided further support for the eQTL findings at the $17 \mathrm{q} 21.32$ locus ${ }^{10}$. This analysis also provided strong evidence of cis-effects for the $17 \mathrm{p} 13.3$ locus lead SNP (rs216172) on the expression of SMG6, (see Supplementary Note and Additional Table 5, see www.imbs-luebeck.de/imbs/sites/default/files/myfilemanager/500/ AdditionalInformation.pdf) ${ }^{10}$. 
We identified five novel loci (9q34, 10q24, 11q23, 15q25, and 17p13) at which the CAD risk variant is fully or strongly correlated $\left(r^{2}>0.8\right)$ with variants that have previously been associated with other traits or diseases ${ }^{11}$. These traits include cerebral and abdominal aneurysm, aortic root size, celiac disease, lung adenocarcinoma, type 1 diabetes, venous thrombosis, LDL cholesterol, HDL cholesterol, triglycerides, smoking, and blood pressure, soluble levels of adhesion molecules, phytosterols (sitosterol and campesterol), angiotensinconverting enzyme (ACE) activity, coagulation factor VIII (FVIII), and von Willebrand factor (vWF) (at $P<5 \times 10^{-8}$, for references see Additional Table 6, see www.imbs-luebeck.de/imbs/sites/default/files/myfilemanager/500/ AdditionalInformation.pdf). Thus, a substantial subset of the novel CAD risk loci appear to have pleiotropic effects. We illustrate a particularly striking example at the $A B O$ locus in Figure 2.

The present genomic analysis of more than 135,000 individuals reveals three major findings. First, we more than doubled the number of loci with firm association to CAD. Specifically, our study yielded 13 novel and confirmed 10 previously reported loci. Second, we found that only a minority of the established and novel loci appear to act through traditional risk factors while the majority resides in gene regions that were not previously suspected in the pathogenesis of CAD. Third, a substantial proportion of the CAD risk variants are also strongly associated with various other human disease traits in GWA studies.

We anticipated that some of the genetic risk loci for CAD would act through established CAD risk factors, such as LDL cholesterol or blood pressure, which themselves have a significant genetic determination. Indeed, three of the novel risk loci (11q23.3, 9q34.2, 10q24.32) showed such associations. An association with higher LDL cholesterol or lipoprotein (a) concentration had also been found for four previously discovered risk variants including the PCSK9 locus that missed genome-wide significance level by a small margin in the present study (Table 1) ${ }^{4,12-13}$. On the other hand, 17 out of the 23 confirmed loci appear to act through mechanisms that are independent of traditional risk factors. Elucidation of these mechanisms is critical for a more complete understanding of CAD and identification of novel therapeutic targets.

We explored several molecular mechanisms by which the novel loci could affect CAD risk. We show that some lead SNPs - or linked variants - affect the primary structure of the protein product in which the variant is located, while in other instances the risk variant is associated with expression of a specific gene or genes in one or more tissues. A more detailed discussion of the genes in each locus is provided in the Supplementary Note. While these data help to prioritize genes for follow-up functional studies, it should be emphasized that substantial work is necessary to define the mechanisms involved for each of the novel loci, as exemplified recently for the chromosome $1 \mathrm{p} 13$ locus $^{14-15}$.

We also observed that eight of 23 CAD loci (five of the 13 novel loci: 9q34, 10q24, 11q23, $15 \mathrm{q} 25$, and $17 \mathrm{p} 13$ and three of the 10 established loci: 1p13, 9p21.3, and 12q24) not only affect the risk of CAD but also associate with multiple other diseases and traits (Additional Table 6, see www.imbs-luebeck.de/imbs/sites/default/files/myfilemanager/500/ AdditionalInformation.pdf). Each of these findings requires further analysis to determine whether co-localization of SNPs for CAD and other traits points to intermediate phenotypes, and thus mechanistic links in a joint etiology, results from pleiotropic effects of a single allele affecting multiple phenotypes, or identifies chromosomal regions harbouring multiple genes and alleles that participate in the regulation of multiple independent traits via diverse mechanisms. 
By design, our study focused on common risk variants. Assuming a heritability of $40 \%$ for $\mathrm{CAD}^{1}$, the lead SNPs of previously established loci combined with the novel loci discovered in this study explain approximately $10 \%$ of the additive genetic variance of CAD. Our inability to explain a greater fraction of CAD heritability even after a large meta-analysis and replication effort is in line with results of most other complex traits examined by current GWA methods ${ }^{16}$. These results suggest that many other common susceptibility variants of similar or lower effects and/or rare variants contribute to risk of CAD.

The clinical utility of CAD risk alleles for the prediction of risk may be best determined in samples that are independent from this discovery study. In order to provide a framework for future research we explored a weighted score based on the 23 CAD risk variants validated in this investigation. We observed a greater than three-fold difference in CAD risk between top and bottom $10 \%$ of the risk scores although this may be a slight overestimation since risk scores were extracted from a subset of the discovery sample (Supplementary Figure 3). Nonetheless, this increase in risk is at least comparable to that of several other traditional risk factors for $\mathrm{CAD}$ including hypertension, diabetes and smoking ${ }^{13}$. Whether risk allele information may improve the performance of current risk profiling strategies for $\mathrm{CAD}$ prediction ${ }^{17-18}$ and whether such an approach is cost-effective requires further evaluation in prospective studies. Our findings provide a firm framework for such research.

In summary, our large-scale GWA meta-analysis discovered the association with CAD of 13 novel chromosomal loci. We observed only limited association between these CAD SNPs and traditional risk factors, suggesting that most SNPs act though novel pathways. Elucidation of the mechanisms by which these loci affect CAD risk carries the potential for better prevention and treatment of this common disease.

\section{Supplementary Material}

Refer to Web version on PubMed Central for supplementary material.

\section{Acknowledgments}

We thank the participants and staff in each of the studies who contributed to the present article. The sources of funding are listed in the supplementary materials.

\section{References}

1. Marenberg ME, Risch N, Berkman LF, Floderus B, de Faire U. Genetic susceptibility to death from coronary heart disease in a study of twins. The New England journal of medicine. 1994; 330:10411046. [PubMed: 8127331]

2. Schunkert H, Erdmann J, Samani NJ. Genetics of myocardial infarction: a progress report. Eur Heart J. 2010; 31:918-925. [PubMed: 20219748]

3. Preuss M, et al. Design of the Coronary Artery Disease Genome-Wide Replication and MetaAnalysis (CARDIoGRAM) Study -- A Genome-Wide Association Meta-Analysis Involving More than 22,000 Cases and 60,000 Controls. Circ Cardiovasc Genet. 2010

4. Clarke R, et al. Genetic variants associated with Lp(a) lipoprotein level and coronary disease. The New England Journal of Medicine. 2009; 361:2518-2528. [PubMed: 20032323]

5. Tregouet DA, et al. Genome-wide haplotype association study identifies the SLC22A3-LPAL2-LPA gene cluster as a risk locus for coronary artery disease. Nature Genetics. 2009; 41:283-285. [PubMed: 19198611]

6. Psaty BM, et al. Cohorts for Heart and Aging Research in Genomic Epidemiology (CHARGE) Consortium: Design of prospective meta-analyses of genome-wide association studies from 5 cohorts. Circ Cardiovasc Genet. 2009; 2:73-80. [PubMed: 20031568] 
7. Nitz I, et al. Association analyses of GIP and GIPR polymorphisms with traits of the metabolic syndrome. Mol Nutr Food Res. 2007; 51:1046-1052. [PubMed: 17624916]

8. Emilsson V, et al. Genetics of gene expression and its effect on disease. Nature. 2008; 452:423-428. [PubMed: 18344981]

9. Zhong $\mathrm{H}$, et al. Liver and adipose expression associated SNPs are enriched for association to type 2 diabetes. PLoS Genet. 2010; 6:e1000932. [PubMed: 20463879]

10. Ge B, et al. Global patterns of cis variation in human cells revealed by high-density allelic expression analysis. Nature Genetics. 2009; 41:1216-1222. [PubMed: 19838192]

11. Hindorff LA, et al. Potential etiologic and functional implications of genome-wide association loci for human diseases and traits. Proc Natl Acad Sci U S A. 2009; 106:9362-9367. [PubMed: 19474294]

12. Brown MS, Goldstein JL. Expression of the familial hypercholesterolemia gene in heterozygotes: mechanism for a dominant disorder in man. Science. 1974; 185:61-63. [PubMed: 4366052]

13. Linsel-Nitschke $P$, et al. Lifelong reduction of LDL-cholesterol related to a common variant in the LDL-receptor gene decreases the risk of coronary artery disease--a Mendelian Randomisation study. PLoS One. 2008; 3:e2986. [PubMed: 18714375]

14. Musunuru K, et al. From noncoding variant to phenotype via SORT1 at the 1p13 cholesterol locus. Nature. 466:714-719. [PubMed: 20686566]

15. Linsel-Nitschke P, Samani NJ, Schunkert H. Sorting out cholesterol and coronary artery disease. The New England Journal of Medicine. 2010; 363:2462-2463. [PubMed: 21158662]

16. Manolio TA, et al. Finding the missing heritability of complex diseases. Nature. 2009; 461:747753. [PubMed: 19812666]

17. Wilson PW, et al. Prediction of coronary heart disease using risk factor categories. Circulation. 1998; 97:1837-1847. [PubMed: 9603539]

18. Ripatti S, et al. A multilocus genetic risk score for coronary heart disease: case-control and prospective cohort analyses. Lancet. 2010; 376:1393-1400. [PubMed: 20971364]

19. Samani NJ, et al. Genomewide association analysis of coronary artery disease. The New England Journal of Medicine. 2007; 357:443-453. [PubMed: 17634449]

20. Gudbjartsson DF, et al. Sequence variants affecting eosinophil numbers associate with asthma and myocardial infarction. Nature Genetics. 2009; 41:342-347. [PubMed: 19198610]

21. Kathiresan $\mathrm{S}$, et al. Common variants at 30 loci contribute to polygenic dyslipidemia. Nature Genetics. 2009; 41:56-65. [PubMed: 19060906] 


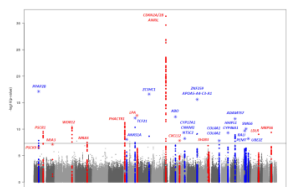

Figure 1. Graphical summary (Manhattan plot) of genome-wide association results The $\mathrm{x}$-axis represents the genome in physical order; the $\mathrm{y}$-axis shows $-\log _{10} \mathrm{P}$ values for all SNPs. Data from the discovery phase are shown in circles and data from the combined discovery and replication phases in stars. Genes at the significant loci are listed above the signals. Known loci are shown in red and novel loci are shown in blue. 


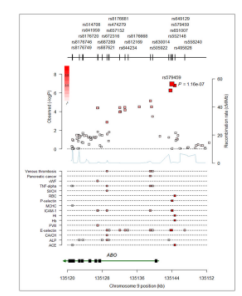

Figure 2. Example of overlapping association signals for multiple traits at $A B O$ gene region on chromosome 9q34

In the upper panel the association signal for coronary disease at the $A B O$ gene region in CARDIoGRAM and the positions and rs-numbers of SNPs in this region are shown. The size of boxes illustrates the number of individuals available for this respective SNP. In the lower panel all SNPs with $P$-values at genome-wide significance level of $P<5 \times 10^{-8}$ based on the NHGRI GWA study catalogue (http://www.genome.gov/gwastudies/; accessed on June $28^{\text {th }} 2010$ ) for all diseases and traits are shown. The degree of linkage disequilibrium $\left(\mathrm{r}^{2}\right)$ between the lead SNPs for coronary disease and the other traits is reflected by the colour of the squares (upper panel) and the small bars (lower panel) (dark red (high LD) $>$ faint red (low LD)). SI/CH = sitosterol normalized to cholesterol; $\mathrm{CA} / \mathrm{CH}=$ campesterol normalized to cholesterol; $\mathrm{ALP}=$ alkaline phosphatase; $\mathrm{ACE}=$ angiotensin converting enzyme; $\mathrm{FVIII}=$ coagulation factor VIII; vWF = von Willebrand Factor. 
.

:. 


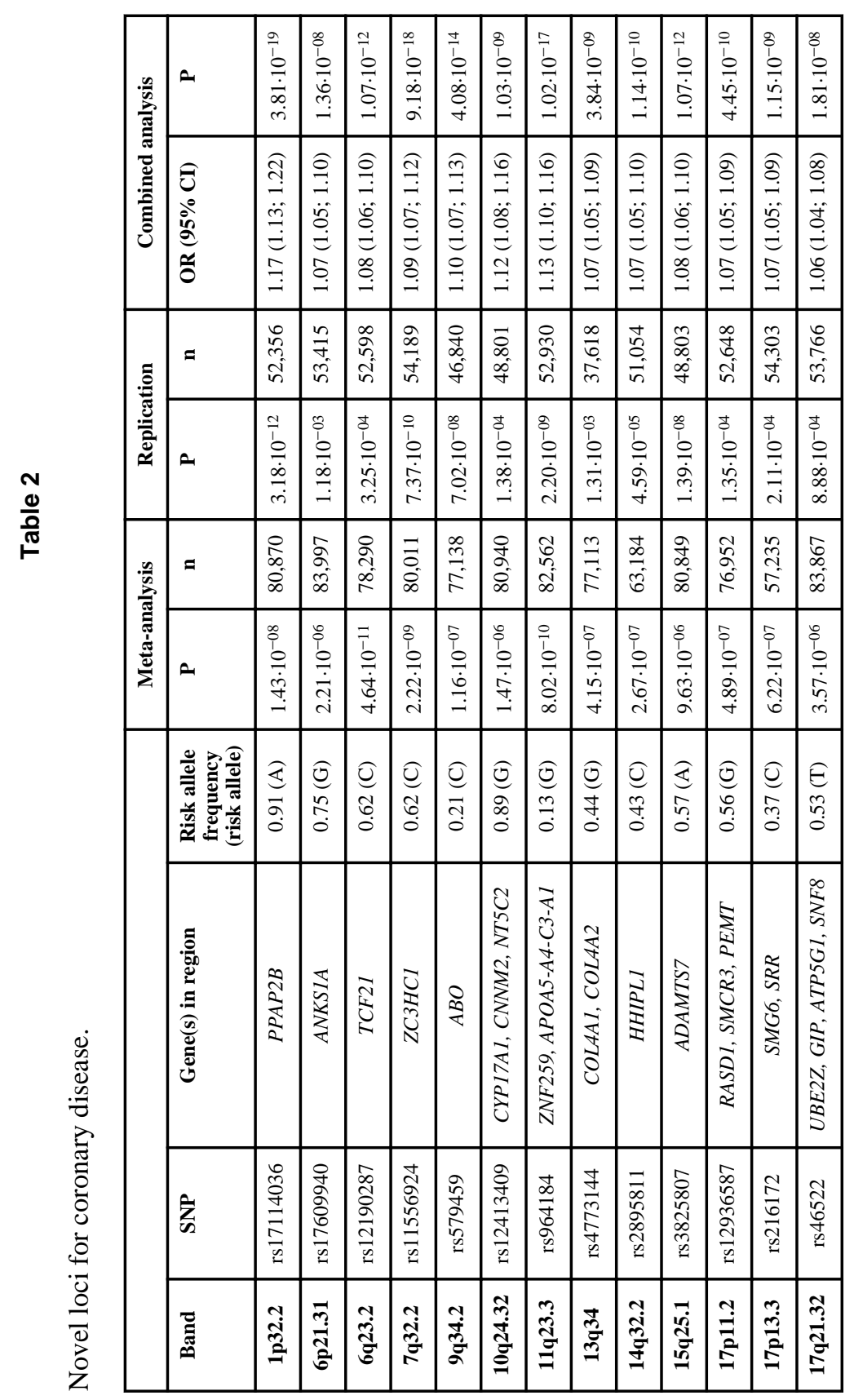




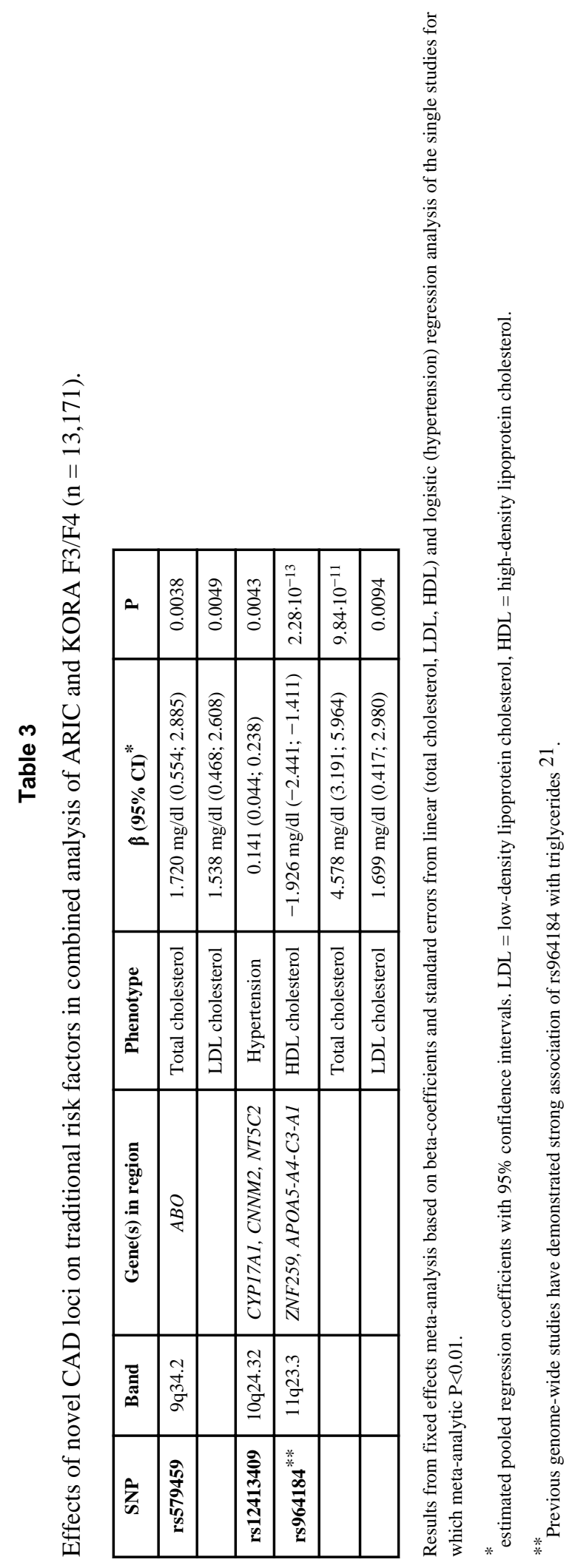

Nat Genet. Author manuscript; available in PMC 2011 October 1. 